\title{
Sitagliptin phosphate
}

National Diabetes Information Clearinghouse (NDIC)

\section{Definitions}

Type 2 diabetes

Defined by National Diabetes Information Clearinghouse (NDIC)

Blood glucose

Defined by National Diabetes Information Clearinghouse (NDIC)

Insulin

Defined by National Diabetes Information Clearinghouse (NDIC)

Liver

Defined by National Diabetes Information Clearinghouse (NDIC)

Glucose

Defined by National Diabetes Information Clearinghouse (NDIC)

DPP-4 inhibitor

Defined by National Diabetes Information Clearinghouse (NDIC)

\section{Source}

National Diabetes Information Clearinghouse (U.S.). (2009). The diabetes dictionary. [Bethesda, Md.]: U.S. Dept. of Health and Human Services, National Institutes of Health, National Institute of Diabetes and Digestive and Kidney Diseases, National Diabetes Information Clearinghouse.

An oral medicine used to treat type 2 diabetes. Sitagliptin lowers blood glucose by helping the body make more insulin when it's needed. It also helps keep the liver from putting stored glucose into the blood. Sitagliptin belongs to the class of medicines called DPP-4 inhibitors. (Brand name: Januvia.) 